\title{
Neurological Implications in the Treatment of Myopia by Means of Orthoculogy
}

John William Yee*

NewGen Optical, 4675 Steeles Avenue East, Toronto, Ontario, Canada

*Corresponding author: John William Yee, 205 Wynford Drive, Unit 2204, Toronto, Ontario, Canada M3C 3P4, Tel: 416-429-3157; Email: johnwilliamyee@gmail.com

Received date: Sep 08, 2014, Accepted date: Dec 09, 2014, Published date: Dec 13, 2014

Copyright: (c) 2014 William Yee J, This is an open-access article distributed under the terms of the Creative Commons Attribution License, which permits unrestricted use, distribution, and reproduction in any medium, provided the original author and source are credited.

\begin{abstract}
Myopia, or nearsightedness, is not just a physical deviation of the eye; there is also a neurological deviation. The incorrect neuromuscular message is sent for distant focusing because the visual cortex interprets the myopic eye to be in near focus mode. When an effort is made to bring a distant object into focus, the crystalline lens will tend to "bulge" instead of becoming "flat", and the eye will tend to elongate instead of remaining spherical. The visual cortex partially or completely overwrites the information received by depth perception: that the object is far away.

The culprit behind this misinterpretation is the spasm of the oblique muscles. How much the neurosensory information is altered depends on the tension of the oblique muscles. If those muscles are partial tight, the visual cortex would send a partially revised neuromotor message; and if those muscles become tighter, the correct neuromotor message for distant focusing would be further revised in favour of near focusing. The premise was demonstrated by applying orthoculogy to treat over 300 patients with myopia. Orthoculogy (or Ortho C) relaxes the oblique muscles to reset the curvature of the crystalline lens and sclera.
\end{abstract}

The following are some indications to demonstrate that the reinstatement of the correct neuromotor message can reverse the effects of myopia:

The ability of ortho $\mathrm{C}$ to treat myopia without direct physical manipulation of the refractive mediums: the crystalline lens, the sclera, or the cornea.

The ability of the visual cortex to discriminate the different treatment options after an ortho $\mathrm{C}$ lens just relaxes the oblique muscles.

The time it takes to restore the mild or moderate myopic eye to its premyopic shape if the intraocular or extraocular muscles are not compromised: in about 2 minutes.

Keywords: Myopia; Orthoculogy; Ortho C; Neurological treatment of myopia

\section{Introduction}

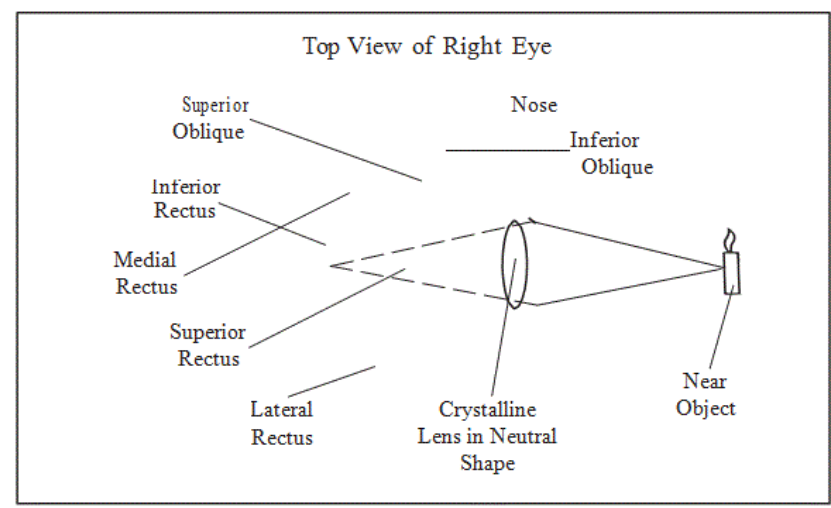

Figure 1: The lens needs to "bulge" to bring a near object into focus.
When the eye becomes myopic, its physical attribute according to conventional optometry is that the eyeball is too long from front to back [1,2]. More specifically, the sclera is too long. Only the outermost part of the eye takes on an elongated shape once it becomes myopic because the innermost part is attached to the optic nerve [3]. But according to Mutti et al. [4], the crystalline lens as well as the sclera becomes myopic during the onset of myopia. This was verified by ultrasound.

In addition, there is a neurological deviation as well as a physical deviation when the eye became myopic. An incorrect neuromotor message is triggered by the spasm of the oblique muscles, and it is relayed to the ciliary and rectus muscles. It maintains the myopic shape of the crystalline lens and sclera [1].

This paper attempts to verify this erroneous message in the myopic eye by:

* Examining the theory of its etiology. It was generated during the onset of myopia. There is a correlation between how the eye became myopic and the spasm of the oblique muscles which altered the correct neuromuscular message.

${ }^{\star}$ Examining how it is possible for orthoculogy (or ortho C, for short) to reverse the shape of the myopic eye by reinstating the correct 
neuromuscular message-by relaxing the spasm of the oblique muscles.

\section{The Theory on the Etiology of the Neurological Deviation}

\section{The Conventional Model}

During close-up work, the focal point is behind the retina due to the divergent rays of light from a near object as oppose to parallel rays when an object is far away. The crystalline lens would have to "bulge" (or become rounder or longer from front to back) to bring it into focus by shifting the focal point forward onto the retina. If the lens did not become rounder, but retained a neutral or "flat" shape instead, the focal point would remain behind the retina as illustrated in Figure 1. (The lens assumes a "flat" or neutral shape to bring a distant object into focus.)

If the lens reaches its maximum "bulged" shape during very closeup work, there would be an increase in "effort to see". The ciliary muscle, which controls the shape of the crystalline lens, would have to tighten up to its maximum before the lens can "bulge" to its maximum. When the ciliary muscle tightens up to that extent, it would expand as much as possible, like how your biceps would expand when you flex them. That causes the zonule fibers around the perimeter of the crystalline lens to ease up. (The fibers attach the lens to the ciliary muscle.) The slack allows the lens to "bulge".

The ciliary muscle can only maintain the maximum "bulged" shape of the lens for so long before fatigue sets in. The extent of the fatigue is represented by the gap between the focal point and the retina when the lens is in a neutral shape as shown in Figure 1. The wider the gap, the more the crystalline lens would have to "bulge" to close it-and more effort would be placed on the ciliary muscle. The eyeball (or sclera) would then have to elongate to alleviate the fatigue.

If the sclera did not participate to relieve some of the fatigue in this manner, then prolonging the fatigue would contribute to asthenopia (or eye strain). The symptom would be in the form of headaches, fatigue, and blurred vision. This is evident in the hyperopic eye where the crystalline lens cannot "bulge" as much [5].

When the oblique muscles tighten up, the axial length of the eye increases. The tighter those muscles become, the greater the elongation. The rectus muscles allow for the lengthening of the sclera by relaxing their tension. The oblique muscles are the vertical extraocular muscles as shown in Figure 1. The rectus muscles are the four lateral extraocular muscles. There are two rectus muscles running across the eye on each side as illustrated in Figure 1.

According to conventional theory, the oblique and rectus muscles do not participate in near or distant focusing, and the sclera does not change its shape during near focusing. The crystalline lens is the only part of the eye that changes its curvature to bring an image into focus [6]. It "flattens" out for distant focusing when the ciliary muscle relaxes. (The ciliary muscle retracts when it relaxes. It causes the zonule fibers to become taut, and the pull "flattens" the lens.) The lens "bulges" for near focusing when the ciliary muscle tightens up. (The tension expands the ciliary muscle. It causes the zonule fibers to become slack, and this easing up of the fibers allows the lens to "bulge".) With sudden changes in focus from near to far and vice versa, only the ciliary muscle can satisfy the need for an immediate change. The crystalline lens can change from near focus mode to distant focus mode and vice versa more readily than the sclera.

The conventional theory of optometry still holds as long as the oblique muscles do not spasm. But once spasm sets in due to near point stress (when performing very close-up work for an extended period of time without taking a break), the tension interrupts the correct neuromotor message sent to the ciliary muscle. It triggers a different model for near and distant focusing [5].

\section{A Different Model}

The ciliary muscle becomes excessively tight if it attempts to maintain the maximum "bulge" of the crystalline lens for an extended period-when the object of regard is very close. The rectus muscles are on standby to allow the shape of the sclera to elongate if necessary to alleviate the tension of the ciliary muscle during prolonged near focusing [5]. The oblique muscles do not just rotate or turn the eye, but they also act as a relay switch to orchestrate the function of the ciliary and rectus muscles for this task. The visual cortex synchronizes the oblique muscles with the ciliary and rectus muscles simultaneously as the tension of the oblique muscles increase. This relationship is possible due to the common neuro-pathway: cranial nerve 3 (or $\mathrm{C}_{3}$ ). The intention is to bring about a possible change in the curvature of the sclera depending on the degree and duration of the tension of the ciliary muscle. This model is activated when the oblique muscles tighten up excessively [1].

If the oblique muscles do not spasm, the ciliary muscle can still relax to "flatten" the lens for distant focusing, and the rectus muscles can still resume their proper tension to maintain the spherical shape of the eye; but once the spasm of the oblique muscles sets in, then the eye is stuck in near focus mode. The spasm of those muscles signals the visual cortex that the ciliary muscle should tense up and the rectus muscles should relax even when the object of regard is in the distance. That is how those muscles would behave to facilitate near focusing under adverse conditions.

The nearsighted eye is actually as an eye that is stuck in near focus mode. If the tension of the oblique muscles of such an eye is permanent, the visual cortex will respond to a distant image differently. The spasm is a signal that the rectus muscles should remain relaxed to allow the sclera to be locked in an oblong shape (by the tension of the oblique muscles) and that the ciliary muscle should remain tense to restrict the crystalline lens in a "bulged" shape.

The neurosensory message from the tension of the oblique muscles takes precedence over the message from depth perception-that the object of regard is in the distance instead of close-up. The spasm of the oblique muscles offsets the correct response to the neurosensory message from a distant object by an amount relative to the degree of tension. If those muscles are partial tight, as in cases of mild myopia, the visual cortex would send a partially revised message; and if those muscles become tighter, as in more severe cases of myopia, the correct neuromotor message for distant focusing would be further modified in favour of near focusing. Although you know that the object is far away, the visual cortex responds by sending a neuromotor message to carry out the function of near focusing [5]. 


\section{Indications of an Incorrect Neuromuscular Message When the Eye Becomes Myopic}

\section{The Impact on the Ciliary Muscle}

The first indication that there is an incorrect neuromuscular message is the dual relationship between the oblique muscles and the ciliary muscle during the onset of myopia. The tension of the oblique muscles is an extension of the tension of the ciliary muscle. It is comparable to the neurological relationship between, for example, the left medial rectus muscle and right lateral rectus muscle when both eyes turn to the right. Both those muscles simultaneously become tense before the eye can turn. This is referred to as Hering's Law [7].

When the spasm of the oblique muscles sets in, the initiator of the stimulation between the ciliary muscle and the oblique muscles would be reversed. The spasm of the oblique muscles would become the determining factor instead of the tension of the ciliary muscle. During the onset of myopia, it was the tension of the ciliary muscle that first influenced the oblique muscles to tighten up (and the rectus muscles to relax to allow for a possible elongation of the sclera). The spasm of the oblique muscles induces a different type of tension onto the ciliary muscle.

The fixed tension of the oblique muscles causes the tension of the ciliary muscle to also become fixed. The spasm of the oblique muscles imposes a limit as to how much the ciliary muscle can "flatten" the crystalline lens for distant focusing.

The restriction placed on the lens, however, is not strictly due to the spasm of the ciliary muscle. The spasm of the oblique muscles does not exactly cause the ciliary muscle to spasm. The spastic tension of the oblique muscles is passed onto the ciliary muscle indirectly. If the spasm was directly induced, the contraction of that muscle would be seized; but the ciliary muscle can still tense up some more for close-up focusing and relax again to a certain extent, depending on the prescription, for distant focusing.

Instead of inducing a spastic tension, the spasm of the oblique muscles neurologically imposes a ceiling on how much the ciliary muscle can relax (and thus how much the lens can "flatten" out) when trying to make something out in the distance. The restriction does not compromise the ciliary muscle's ability to further tighten up to allow the crystalline lens to "bulge" some more for near focusing. During early onset, the lens can still "flatten" (from its "bulge" shape when focusing on something close-up) to bring a distant object into focus.

\section{The Impact on the Rectus Muscles}

The second indication that there is an incorrect neuromuscular message is when the mild myopic eye progresses to moderate, midrange, or severe myopia. At these stages, the sclera as well as the crystalline lens is affected. The modified message is relayed to the rectus muscles via cranial nerve 3 (or $\mathrm{C} 3$ ) once the visual cortex picks up the modified neurosensory message (the tension of the oblique muscles). The rectus muscles would relax accordingly to allow the sclera to elongate.

The spasm of the oblique muscles also creates a dual relationship between the oblique muscles and rectus muscles. It is comparable to the neurological relationship between the medial rectus muscle and lateral rectus muscle, for example, when the eye turns to one side: one muscle must relax to allow the tension of the other muscle to turn the eye in that direction. This is referred to as Sherrington's Law [7]. Perhaps the spasm of the oblique muscles activates a similar relationship with the rectus muscles during developmental myopia.

\section{The Synchronization of the Two Dual Relationships}

After the rectus muscles relax, the visual cortex simultaneously relays the modified neuromotor message to the ciliary muscle also via cranial nerve 3 . The ciliary muscle would tighten up accordingly to restrict the crystalline lens to a predetermined "bulged" shape instead of allowing it to "flatten" for distant focusing [5]. The synchronization of the tension of the ciliary muscle and the relaxation of the rectus muscles would promote near focusing even when the object or regard is in the distance. The synchronization of the ciliary muscle and rectus muscles is due to the synchronization of the two dual relationships between the oblique muscles and ciliary muscle and the oblique muscles and rectus muscles. The synchronization of these two dual relationships is initiated by the spasm of the oblique muscles in the higher myopic ranges.

\section{Indications That the Correct Neuromotor Message Can Reverse the Effects of Myopia}

\section{Treating Myopia without Physically Manipulating the Lens, Sclera, or Cornea}

The transmission of an incorrect neuromuscular message can be demonstrated by orthoculogy. The treatment involves relaxing the oblique muscles to reverse the myopic shape of the eye by reinstating the correct neuromuscular message. This is accomplished by wearing a contact lens designed to relieving the spasm of the oblique muscles [1]. The theory that the spasm of the oblique muscles is responsible for short circuiting the correct neurological message has to be valid before ortho $\mathrm{C}$ can work since ortho $\mathrm{C}$ targets just the spasm of the oblique muscles instead of physically altering the shape of the lens, sclera, or the cornea. The ability to "correct" or "reduce" the refractive error by relaxing just the oblique muscles for a few minutes without directly tampering with any refractive medium suggests that the spasm of those muscles initiated the neurological error-and that it is possible to offset it by reinstating the correct neuromuscular message.

If somehow the correct message was able to be sent without the assistance of ortho $\mathrm{C}$ during early onset, the myopic shape of the crystalline lens would have reverted back to the correct shape for distant focusing just as easily and just as quickly. Only the lens was affected during the initial stage, and the intraocular and extraocular muscles would not have been compromised. This sometimes occurs in cases of pseudomyopia [8]. Perhaps the spasm of the oblique muscles occurs intermittently, and developmental myopia has not been established yet. A recovery in visual acuity is possible when those muscles can completely relax.

\section{Treating Mild Myopia}

In most cases, mild myopia is mainly due to the "bulged" shape of the crystalline lens. It cannot "flatten" out sufficiently for distant focusing. Mild myopia sets in when the oblique muscles became permanently tight but not so tight that it forces the sclera to elongate [5]. The treatment for mild myopia involves only the crystalline lens. By reinstating the correct neuromuscular message, it resets the proper curvature of the lens. It demonstrates that a neurological relationship 
exists between the oblique muscles and ciliary muscle-and thus the crystalline lens [5].

Unlike the relationship between the crystalline lens and the ciliary muscle, there is no direct connection between the oblique muscles and the ciliary muscle. You cannot mechanically trace how the oblique muscles "flatten" the crystalline lens. The relationship between the oblique muscles and the crystalline lens do not have a physiological link between them like the relationship between the ciliary muscle and the crystalline lens. And yet, the spasm of the oblique muscles can lock in the tension of the ciliary muscle-and thus the myopic shape of the crystalline lens.

When ortho $\mathrm{C}$ restores proper focusing by relaxing the oblique muscles slightly with a type 1 lens, it indirectly relaxes the ciliary muscles. A type 1 lens just relaxes the oblique muscle muscles sufficiently to entice the ciliary muscle to relax and thereby corrects mild myopia by "flattening" out the crystalline lens for distant focusing. It does not relax the oblique muscles to the extent that it stimulates the sclera to "shift". If a type 2 lens forced the sclera to "shift" instead of the crystalline lens, even by a minimal amount, the treatment would fail. There would still be an incorrect message affecting the crystalline lens [5]. This simple test, if it was carried out, would indicate that it is necessary to target the treatment to the part of the eye affected by the incorrect neurological message. By indiscriminately altering the part of the eye that is unaffected, it would not offset the incorrect neurological message.

When a type 1 lens relaxes the tension of the ciliary muscle by relaxing the oblique muscles, it suggests that the transmission of the correct neuromotor message was initiated by the spasm of the oblique muscles. The reception of a new neurosensory message (that the oblique muscles are relaxed instead of being tense for distant focusing) stimulates the delivery of the proper neuromotor message for distant focusing.

\section{Treating Severe Myopia}

When a type 2 lens reduces severe myopia, it demonstrates that a neurological relationship exists between the oblique muscles and the rectus muscles. By reinstating the correct neuromuscular message, it attempts to reset the proper curvature of the sclera. In the short term, ortho C can only "reduce" severe myopia instead of "correcting" it as in mild or moderate myopia. The intraocular and extraocular muscles are compromised in severe myopia. Thus it is necessary to also implement physiotherapy as part of the long term treatment by wearing the lenses more frequently. But the short term result, which is indicative of a neurological effect, can still be measured. If the eye is receptive to ortho $\mathrm{C}$, a 2 or 3 line improvement can be seen on the Snellen chart after the first application when the patient wears the lenses for 2 minutes.

A type 2 lens can stimulating the ciliary muscle to maintain the relaxation acquired from a type 1 lens and thus maintain the improved shape of the crystalline lens. A type 2 lens can also "loosen" the oblique muscles to the extent of allow the rectus muscles to tighten in order to reduce the elongated shape of the sclera. It is the reverse of how those muscles were affected when the oblique muscles became tense. There is no connection between the oblique muscles and the rectus muscles, but the oblique muscles can still stimulate the rectus muscles to tighten up.

When a type 2 lens increases the tension of the rectus muscles by relaxing the oblique muscles, it suggests that the transmission of the correct neuromotor message was initiated by the spasm of the oblique muscles. The reception of a new neurosensory message (that the oblique muscles are relaxed instead of being tense for distant focusing) stimulates the delivery of the proper neuromotor message for distant focusing.

\section{The Ability to Discriminate Among the Different Types of Treatment}

In some cases, it is possible to treat different types of refractive errors with an ortho $\mathrm{C}$ lens of a similar design. A type 1 lens, for example, is designed only to relax the oblique muscles just enough to stimulate the ciliary muscle to relax. The intention is to alter the curvature of the crystalline lens without causing the sclera to "shift". The design of this lens can be applied to correct mild myopia or the different types of simple astigmatism. If the lens was intended to treat simple lenticular astigmatism, for example, the visual cortex can distinguish that specific problem from other possible problems that may arise if only the ciliary muscle (and thus the crystalline lens) was affected by the spasm of the oblique muscles. The visual cortex did not base that identification on the design of the lens.

The visual cortex can still deliver the proper neurological message for that specific problem despite the different ways the crystalline lens may be altered given the range of possible problems. To correct mild myopia, the ortho $\mathrm{C}$ contact lens only needs to "stretch" the oblique muscles sufficiently to allow the crystalline lens to uniformly "flatten" out for distant focusing [1]. To correct corneal astigmatism, the ortho C contact lens only needs to "stretch" the oblique muscles sufficiently to allow the crystalline lens to compensate for the distortion of the cornea along a specific meridian [9]. To correct lenticular astigmatism, the ortho C contact lens only needs to "stretch" the oblique muscles sufficiently to allow the crystalline lens to "flatten" along a specific meridian for distant focusing [10].

\section{References}

1. Yee J (2011) Correcting mild myopia by means of orthoculogy. Med Hypotheses 76: 332-335.

2. Grierson I (2000) The eye book. Liverpool, England: Liverpool University Press.

3. Kaufman A, Alm A (2003) Adlera $^{\text {Tx }}$ s physiology of the eye. St. Louis, Missouri: Mosby Incorporated.

4. Mutti DO, Mitchell GL, Sinnott LT, Jones-Jordan LA, Moeschberger ML, et al. (2012) Corneal and crystalline lens dimensions before and after myopia onset. Optom Vis Sci 89: 251-262.

5. Yee JW (2013) Preventing retinal detachment by averting asthenopia that contributes to progressive myopia. OA Medical Hypothesis 1: 15.

6. Cahan D (1993) Hermann von helmholtz and the foundations of nineteenth-century science. Berkeley, LA: University of California Press.

7. Chornell G, Hanebaum U, Hauck K, Jeffries D, Lorimer B, et al. (2010) Advanced practise 2: Sight testing. Edmonton, AB: Northern Alberta Institute of Technology.

8. Sato T (1957) The causes of acquired myopia. Toyko: Kanehara Shuppan.

9. Yee JW (2012) Correcting corneal astigmatism by reinstating the correct neuromuscular message. See comment in PubMed Commons below Med Hypotheses 79: 368-371.

10. Yee JW (2013) Correcting lenticular astigmatism by reinstating the correct neuromuscular message. See comment in PubMed Commons below Med Hypotheses 81: 36-40. 
Citation: William YJ (2014) Neurological Implications in the Treatment of Myopia by Means of Orthoculogy. J Neurol Neurophysiol 5: 257. doi: 10.4172/2155-9562.1000257 